\title{
Primeira semana saúde integral do recém-nascido: ações de enfermeiros da Estratégia Saúde da Família
}

omo citar este artigo: Lucena DBA, Guedes ATA, Cruz TMAV, Santos NCCB, Collet N, Reichert APS. Primeira semana saúde integral do recém-nascido: ações de enfermeiros da Estratégia Saúde da Família. Rev Gaúcha Enferm. 2018;39:e2017-0068. doi: http://dx.doi.org/10.1590/19831447.2018.2017-0068.
Universidade Federal da Paraíba (UFPB). João Pessoa Paraíba, Brasil.

Universidade Federal de Campina Grande (UFCG) Campina Grande, Paraíba, Brasil.
Daniele Beltrão de Araújo Lucenaa Anna Tereza Alves Guedes ${ }^{a}$

Tarciane Marinho Albuquerque de Vasconcellos Cruz ${ }^{\mathrm{a}}$ Nathanielly Cristina Carvalho de Brito Santos ${ }^{b}$

Neusa Collet ${ }^{a}$

Altamira Pereira da Silva Reichert ${ }^{\mathrm{a}}$

RESUMO

Objetivo: Descrever as ações de enfermeiros da Estratégia Saúde da Família acerca da Primeira Semana Saúde Integral no cuidado ao recém-nascido.

Método: Pesquisa descritiva, exploratória com abordagem qualitativa realizada de outubro de 2014 a fevereiro de 2015, por meio de entrevista semiestruturada, com nove enfermeiros da Estratégia Saúde da Família de João Pessoa-PB. Os dados foram submetidos à análise temática.

Resultados: As ações identificadas na primeira visita ao bebê se baseiam nas orientações maternas acerca dos cuidados básicos ao recém-nascido, aleitamento materno, testes de triagem neonatal, imunização e puericultura, bem como avaliação da puérpera, no entanto, por vezes eram realizadas fora do período recomendado e com orientações incompletas e desatualizadas.

Conclusão: Embora haja potencialidades nas ações dos enfermeiros prestadas a essa população, as fragilidades comprometem a assistência ao neonato e à puérpera, sendo necessário sensibilizar esses profissionais acerca da importância e eficácia da Primeira Semana Saúde Integral.

Palavras-chave: Recém-nascido. Atenção primária à saúde. Visita domiciliar. Enfermagem.

\section{ABSTRACT}

Objective: To describe the nurses' actions of the Family Health Strategy about the First Week for Integral Health regarding the care devoted to the newborn

Method: It is a descriptive, exploratory research with qualitative approach carried out from October 2014 to February 2015, through a semi-structured interview, with nine nurses from the Family Health Strategy of João Pessoa-PB. Data were submitted to thematic analysis. Results: The actions identified at the first visit to the newborn child are based on maternal guidance on basic newborn care, breastfeeding, neonatal screening, immunization and childcare, as well as evaluation of the puerperal, but it was sometimes performed outside the period recommended and with incomplete and outdated guidelines.

Conclusion: Although there are potentialities in nurses'actions to this population, the fragilities compromise the care of the newborn and the puerperium, and it is necessary to sensitize these professionals about the importance and effectiveness of First Week for Integral Health. Keywords: Infant, newborn. Primary health care. Home visit. Nursing.

\section{RESUMEN}

Objetivo: Describir las acciones de enfermeros de la Estrategia Salud de la Familia acerca de la Primera Semana Salud Integral en la atención al recién-nacido.

Método: Investigación descriptiva, exploratoria con abordaje calitativo, realizada de octubre de 2014 hacia febrero de 2015, por medio de entrevista semiestructurada, con nueve enfermeros de la Estrategia Salud de la Familia de João Pessoa-PB. Los datos fueron sometidos al análisis temático.

Resultados: Las acciones identificadas en la primera visita al bebé se basan en las orientaciones maternas sobre los cuidados básicos al recién nacido, la lactancia materna, las pruebas de selección neonatal, la inmunización y la puericultura, así como la evaluación de la puérpera, pero a veces se realizaban fuera del período recomendado y con directrices incompletas y obsoletas.

Conclusión: Aunque hay potencialidades en las acciones de los enfermeros prestadas a esa población, las fragilidades comprometen la asistencia al neonato y a la puérpera, siendo necesario sensibilizar a esos profesionales acerca de la importancia y eficacia de la Primera Semana Salud Integral.

Palabras clave: Recién nacido. Atención primaria de salud. Visita domiciliaria. Enfermería 


\section{—INTRODUÇÃO}

Todo ano mais de 3 milhões de recém-nascidos (RN) vão a óbito antes do primeiro mês de vida, dos quais três quartos morrem na primeira semana e um terço não sobrevive ao primeiro dia de vida (1). Considerando isso, a Organização das Nações Unidas (ONU) propôs a criação dos Objetivos de Desenvolvimento do Milênio (ODM), sendo um deles a redução em dois terços da mortalidade infantil até o ano de $2015^{(2)}$.

Os indicadores dos ODM demonstram que, no período de 1990 a 2013, o Brasil reduziu a mortalidade em menores de um ano de 51,4 óbitos a cada mil nascidos vivos para 12,3 óbitos ${ }^{(3)}$, comprovando uma melhoria na atenção à saúde. No entanto, apesar do progresso, ainda há um longo caminho a percorrer para que haja uma maior redução dos indicadores de mortalidade infantil.

No que concerne à mortalidade neonatal (0-27 dias), dados de 2011 a 2012 indicam que o Brasil apresenta uma média de 11,1 óbitos para cada mil nascidos vivos. Destaca-se, ainda, que essas taxas são mais expressivas quando relacionadas as primeiras 24 horas de vida(4).

Os óbitos neonatais geralmente estão associados a uma falha na assistência prestada às mães e aos RN durante $\mathrm{O}$ pré e pós-parto, sendo estes considerados evitáveis quando os agravos são detectados precocemente ${ }^{(1)}$. Nesse sentido, o Ministério da Saúde (MS), tendo por base a Organização Mundial de Saúde (OMS) e o Fundo das Nações Unidas para a Infância e a Adolescência (UNICEF), desenvolveu uma capacitação para profissionais da saúde em Atenção Integrada às Doenças Prevalentes na Infância (AIDPI) com o intuito de promover uma rápida e significativa redução da mortalidade na infância ${ }^{(5)}$. Seguindo ainda a necessidade de que o acompanhamento do RN e da puérpera deve ser iniciado o quanto antes, a fim de avaliar as condições de saúde do binômio mãe-bebê, o MS preconizou a "Agenda de Compromissos para Saúde Integral da Criança e Redução da Mortalidade Infantil" que consiste em recomendações para a assistência à criança por meio de linhas de cuidado, sendo uma delas a Primeira Semana Saúde Integral (PSSI)(5). Ainda em se tratando da iniciativa do governo em melhorar a saúde dessa população, em 2015, por meio da Portaria no 1.130, o MS instituiu a Política Nacional de Atenção Integral à Saúde da Criança (PNAISC) com o objetivo de promover e proteger a saúde da criança e o aleitamento materno ${ }^{(6)}$. Assim, percebese que as autoridades têm-se mostrado interessadas não só em reduzir a mortalidade infantil, mas também em promover qualidade de vida às crianças.

A PSSI é um protocolo de atenção que visa possibilitar cuidado integral e multiprofissional à puérpera e ao neonato na primeira semana após o parto, com a finalidade de identificar sinais de risco que possam comprometer o crescimento e desenvolvimento saudável do RN, orientar as puérperas acerca dos cuidados com o mesmo, incentivar o aleitamento materno, oferecer apoio às dificuldades apresentadas, verificar e aprazar as vacinas e agendar a consulta de puericultura, contribuindo, assim, para a redução da morbimortalidade infantil(5).

Logo, as ações preconizadas para a PSSI devem fazer parte da rotina dos profissionais da Atenção Primária à Saúde (APS), no sentido de realizar uma assistência integral e individualizada, atendendo aos pressupostos da atenção básica de prevenção de doenças, promoção e recuperação da saúde.

Um dos métodos indicados para a execução dessa linha de cuidado se encontra nas visitas domiciliares na primeira semana de vida do bebê ${ }^{(7)}$, que tem eficácia comprovada na redução da mortalidade neonatal ${ }^{(8)}$. No entanto, os esforços dirigidos para a implementação da visita domiciliar no Brasil ainda são falhos, como constata um estudo realizado no Rio de Janeiro(9) no qual apenas 52\% dos atendimentos aos RN ocorreram na primeira semana, quando deveria ter sido abrangente a todos os neonatos, segundo preconiza a PSSI. Dessa forma, apesar de já haver meios para efetuar uma mudança no modelo assistencial, ainda observa-se continuidade do modelo de assistência curativista em detrimento a uma assistência integral à saúde.

Assim, tendo em vista que o enfermeiro é o profissional que se encontra na primeira linha de cuidado prestado à essa população e que sua vivência da PSSI influencia na prevenção e promoção da saúde do RN e, consequentemente, a mortalidade neonatal, justifica-se esse estudo pela necessidade de se investigar como está ocorrendo a atenção às crianças na primeira semana de vida, no contexto da APS. Tal inquietação conduziu à seguinte questão norteadora: Quais as ações desenvolvidas pelos enfermeiros na Primeira Semana Saúde Integral no âmbito da Estratégia Saúde da Família durante a visita domiciliar ao RN? Portanto, o presente estudo tem como objetivo descrever as ações de enfermeiros da ESF acerca da Primeira Semana Saúde Integral no cuidado ao RN.

\section{— MÉTODO}

Trata-se de um estudo descritivo exploratório com abordagem qualitativa que é um método que estuda a história, as relações, as representações, as crenças e as percepções que os sujeitos fazem de si mesmos e de como vivem, sentem e pensam ${ }^{(10)}$. Este estudo é subprojeto de uma pesquisa intitulada "Primeira Semana Saúde Integral: construção e validação de um instrumento para a visita domiciliar ao RN", aprovado pelo CNPq sob o número 447572/2014-8. 
O estudo foi realizado no período de outubro de 2014 a fevereiro de 2015 no município de João Pessoa - PB, que é dividido em cinco Distritos Sanitários contando com 184 Equipes de Saúde da Família (ESF) no total, no qual houve, no ano de 2015, 12.299 nascimentos. O cenário da pesquisa se passa no Distrito Sanitário III devido ao fato de estar localizado na área de abrangência da Universidade Federal da Paraíba (UFPB) e caracterizar-se como cenário de prática dos cursos de graduação desta instituição, conta com 53 ESF e seus respectivos 53 enfermeiros que são responsáveis pela assistência à saúde de 90,5\% das famílias daquela área.

Os participantes da pesquisa foram 9 enfermeiros escoIhidos de forma aleatória e de acordo com sua disponibilidade, mas que atenderam ao seguinte critério de inclusão: ser enfermeiro atuante nas ESF há pelo menos um ano e estar em atividade no momento da coleta de dados.

Para viabilizar a coleta de dados foi utilizado um roteiro semiestruturado com a seguinte questão norteadora: Quais as ações que você realiza durante a primeira visita domiciliar ao RN?

As entrevistas foram realizadas pela pesquisadora, gravadas em mídia digital e com duração média de 15 minutos, sendo realizadas nas Unidades de Saúde da Família (USF) em um ambiente que proporcionasse privacidade e silêncio. Nos resultados, cada enfermeiro foi representado pela letra "E" seguida do numeral em ordem crescente.

O encerramento da coleta ocorreu quando não houve acréscimo de novas informações pertinentes aos objetivos com a continuidade das entrevistas ${ }^{(10)}$. Dessa forma, a coleta dos dados foi encerrada em nove entrevistas, quando houve saturação teórica dos discursos acerca da temática.

A análise dos dados foi feita por meio da técnica de análise temática ${ }^{(10)}$. Inicialmente foi feita uma leitura flutuante e exaustiva das respostas dos entrevistados para a construção dos núcleos de sentido. Em seguida, as ideias centrais foram extraídas das falas conforme os questionamentos e agrupadas por semelhança temática. Posteriormente, uma nova leitura foi feita a fim de organizar o material de acordo com os tópicos que os entrevistados destacaram, sendo agrupados em categorias e discutidas à luz da literatura nacional e internacional ${ }^{(10)}$.

Todos os participantes assinaram o Termo de Consentimento Livre e Esclarecido após conhecimento do objetivo do estudo conforme as diretrizes e normas regulamentadoras para pesquisa envolvendo seres humanos, estabelecidas na Resolução no. 466/12 do Conselho Nacional de Saúde. A pesquisa foi aprovada pelo comitê de ética do Centro de Ciências da Saúde da Universidade Federal da Paraíba, sob o protocolo no 008/15, CAAE: 39801714.2.0000.5188.

\section{— RESULTADOS E DISCUSSÃO}

Participaram desse estudo nove enfermeiros dos quais apenas um pertencia ao sexo masculino. Dois enfermeiros tinham menos de 10 anos de formados, enquanto sete tinham entre 20-35 anos de graduação, assim, a maioria dos entrevistados possui bastante experiência prática na atenção básica, e possivelmente vivenciaram as transições que vem ocorrendo no modelo assistencial ao longo dos anos. Isso pode justificar uma grande busca por cursos, especializações e capacitações, sendo assim, todos afirmaram ter ao menos uma especialização, sendo nas áreas de: Saúde da Família, Saúde Pública, Saúde Coletiva, Programa Saúde da Família (PSF) e Projeto de Profissionalização dos TrabaIhadores da Área de Enfermagem (PROFAE).

A partir da análise dos dados empíricos foi construída a seguinte categoria: Ações do Enfermeiro na Primeira Semana Saúde Integral.

\section{Ações do Enfermeiro na Primeira Semana Saúde Integral}

A realização das ações conforme a PSSI pelo enfermeiro são relevantes devido ao potencial de redução da mortalidade neonatal e melhoria da qualidade de vida do binômio mãe-bebê. A implementação destas auxiliam no empoderamento materno quanto ao autocuidado e cuidados ao RN. Nesse sentido, a primeira ação da PSSI relatada pelos enfermeiros diz respeito à periodicidade da visita domiciliar ao neonato e à puérpera.

A gente sempre tenta priorizar essa visita domiciliar na primeira semana porque é quando assim que ela chega da maternidade para as primeiras orientações. (E1)

Com 15 dias né, a gente faz a visita puerperal da mãe. Quando chega aí o agente de saúde nos comunica que tem o bebê, a gente vai e faz a visita em casa. (E4)

O certo mesmo é ir na primeira semana que tem no livro da criança na página 17. (E5)

A primeira consulta ou visita puerperal nos primeiros 15 dias, não na primeira semana [...] a primeira semana a gente geralmente não visita. (E8)

Percebe-se nos relatos que há discrepância nos discursos, uma vez que há profissionais que realizam a visita domiciliar no tempo correto, no entanto, há também aqueles que descumprem o tempo preconizado. Considerando 
que a realização da visita domiciliar precocemente é uma estratégia para fornecer intervenções sobre os cuidados básicos com o RN e promover a saúde deste, quando essa não é realizada durante o período recomendado, que segundo o MS deve ocorrer entre o terceiro e quinto dia após o nascimento, interrompe algumas ações como auxiliar o desenvolvimento da parentalidade, melhorar o desenvolvimento cognitivo de crianças de baixo-peso ou prematuros, reduzir a ocorrência de lesões não intencionais, melhorar a detecção precoce de depressão pós-parto e prolongar a amamentação(7), ou seja, reduz o potencial da vigilância à saúde e produz impacto na qualidade de vida e na mortalidade dessa população.

Vale destacar que, dentre as ações essenciais da APS, a visita domiciliar é uma estratégia potente por possibilitar uma compreensão ampliada do processo saúde-doença e proporcionar intervenções relacionadas ao cuidado e à manutenção da saúde, tanto do indivíduo quanto da família, perpassando o limite das práticas curativas ${ }^{(11)}$.

Estudos realizados na Malásia, Nepal, Bangladesh e Uganda ${ }^{(8)}$ identificaram que após um período de implementação da visita domiciliar na primeira semana de vida do bebê houve melhoria da saúde deste e redução da mortalidade neonatal.

Entretanto, alguns enfermeiros pontuaram duas razões com o intuito de justificar o adiamento da visita domiciliar ao RN e à puérpera: a primeira, relativa ao agendamento pelo Agente Comunitário de Saúde (ACS) após o retorno da puérpera da maternidade, e a segunda, ao fato de que puérperas submetidas à cesariana costumam não retornar ao seu domicílio com sete dias, ou ainda, que as mesmas costumam passar o período puerperal na casa da mãe ou de familiares.

Para o agente de saúde estar atento a essa chegada, às vezes eu nem sei, quando eu vou saber já tá quinze dias, vinte dias que essa mãe chegou, ou seja, o agente de saúde tem que estar atento se tem tantas gestantes, em qual o período que essa gestante vai estar parindo, tendo seu bebê, chegando em casa para me avisar. (E2)

A (gestante) que pariu por último eu ainda não tive condições de vê-la, eu fui justamente com sete dias e ela não se encontrava, tinha ido fazer o teste do pezinho porque o ACS muitas vezes marca e esquece de olhar as marcações que o hospital, a própria maternidade fez, eles já fazem um agendamento, aí já tive essa dificuldade. (E3)

Eu sempre falo para os agentes de saúde quando nascer diga logo para a primeira visita ser na semana seguinte. (E5)
Quando ela (a puérpera) não está ou quando ela vai para a casa de um parente, então a gente tem um pouco de distanciamento nesse sentido. (E1)

Quando elas saem da maternidade elas procuram ou ir para casa da mãe, ou para a casa da sogra, ou de um familiar, né? Quando acontece isso a gente não consegue captar o recém-nascido na primeira semana, muitas vezes, a gente só consegue captar já perto dos 45 dias, quando elas retornam para casa. (E3)

Foi possível identificar que os enfermeiros da ESF atribuem a captação da mãe no puerpério e o agendamento da visita domiciliar ao ACS, entretanto, o enfermeiro deve ter um controle da quantidade de gestantes e das datas prováveis do parto, a fim de planejar, junto a equipe, a agenda das visitas ao binômio mãe-bebê e sua família. Ademais, a busca ativa das puérperas é de responsabilidade de toda a ESF, assim, não se justifica o atraso da visita domiciliar, bem como a enfermeira não está isenta de responsabilidade pela demora da captação ou marcação pelo ACS uma vez que é sua atribuição a coordenação destes.

Em se tratando do deslocamento das puérperas para áreas fora da abrangência da equipe de saúde relatado neste estudo, condiz com a realidade local e é bastante preocupante uma vez que leva a um comprometimento das ações da equipe para a realização das orientações em tempo ideal, oportuno e com impacto direto na efetividade da PSSI, estando em conformidade com estudo ${ }^{\text {(12) }}$ realizado no município de Teresina (PI) que também apontou a inviabilização do atendimento nos primeiros dias do puerpério devido deslocamento da mãe.

Percebe-se, portanto, que apesar da visita domiciliar ser efetiva para a redução da mortalidade infantil, demonstrada por estudos internacionais, a literatura nacional bem como o presente estudo identificou que os profissionais encontram dificuldades em seguir o que é preconizado, emergindo, assim, a necessidade de melhor compreender que outros fatores influenciam negativamente na realização da visita.

Com o intuito de tentar garantir que as puérperas que não retornarão para seu domicílio logo após o parto tenham as orientações necessárias para cuidar do bebê e garantir o aleitamento materno, um dos enfermeiros orienta as gestantes no pré-natal acerca desses cuidados, uma vez que a visita domiciliar não poderá ser realizada no período recomendado.

Quando ela me avisa: "assim que eu ganhar, eu vou para a casa da minha mãe e vou passar tantos dias e só volto 
tal dia", eu sempre passo as informações para ela no prénatal. (E6)

Compreende-se que é possível fazer as orientações quanto aos cuidados com o RN, ainda no pré-natal, como forma de empoderar o cuidado materno qualificado no período neonatal, mesmo diante da impossibilidade da visita na primeira semana. Este aspecto é corroborado por estudos que afirmam a importância das ações de educação em saúde no pré-natal com orientações sobre os cuidados com o RN para empoderar a mãe e ajudá-la a ser independente no cuidado ao filho ${ }^{(13)}$.

Todavia, há aspectos que transcendem o alcance de orientações prévias e que somente durante a avaliação do RN podem ser detectados, sobretudo no que se refere aos principais sinais que indicam a necessidade de encaminhamento de urgência (recusa alimentar, vômitos importantes, convulsões ou apneia, frequência cardíaca abaixo de 100bpm, letargia ou inconsciência, respiração rápida (acima de 60 irpm), atividade reduzida, febre $\left(>37,5^{\circ} \mathrm{C}\right)$, hipotermia, tiragem subcostal, batimentos da asa do nariz, cianose generalizada ou palidez importante, icterícia visível, fontanela abaulada, secreção purulenta do ouvido, umbigo hiperemiado, pústulas na pele, irritabilidade e dor à manipulação) e na detecção de possíveis fatores de riscos ambientais que tornam imprescindíveis o acompanhamento do profissional em domicílio durante os primeiros dias de vida ${ }^{(5,7)}$.

No sentido de garantir a integralidade do cuidado, os participantes deste estudo afirmaram que a primeira intervenção após o nascimento, no domicílio, é focada na prestação de cuidados básicos ao RN e na maneira que eles estão sendo realizados pela mãe:

Olho o coto umbilical, oriento também a dar banho de sol, vejo se fez o teste do pezinho, o teste do olhinho, vejo se iniciou as vacinas, a gente observa também se está com icterícia, se está amarelinho, se está mamando direitinho, orienta também essa questão de colocar sempre de lado para não regurgitar, não broncoaspirar. (E4)

Oriento as puérperas, vejo como é que está o umbiguinho dele, se está bem, se está bem cicatrizado, se está procedendo direitinho colocando álcool, a higienização dele, a limpeza da boquinha dele, o mamar, o amamentar para não ferir o bico do peito da mãe, a posição e também sobre arroto de colocar em pé e no dormir. (E9)

Esses relatos revelam a preocupação dos enfermeiros em avaliar e orientar os cuidados necessários ao neonato, no entanto, ações como a realização do exame físico completo não foram citadas nos discursos, assim como as orientações foram superficiais e incompletas, além de ter havido recomendações defasadas a exemplo do posicionamento lateral para evitar broncoaspiração, uma vez que as diretrizes atuais são para a posição dorsal ou supina com o intuito de prevenir a morte súbita do lactente ${ }^{(7)}$.

Os países em desenvolvimento têm demonstrado que há pouca cobertura dos cuidados no período pós-parto. Diante disso, ressalta-se a relevância de haver uma maior preocupação relacionada às necessidades básicas do neonato em domicílio, já que este período envolve desafios físicos, emocionais e sociais à mãe e à criança ${ }^{(14)}$ interferindo, também, na qualidade de vida de ambos.

Durante a visita na primeira semana de vida do bebê, cabe ao enfermeiro esclarecer dúvidas e abordar cuidados específicos ao RN, buscando orientar sobre a higiene bucal, sono e repouso, cuidados com o coto umbilical, amamentação, banho, troca de fraldas, agasalhamento, prevenção de assaduras, banho de sol, realização dos testes de triagem neonatal (teste do pezinho e teste da orelhinha), relações familiares, importância das vacinas, orientação quanto ao seguimento do RN nas consultas de puericultura, bem como o planejamento familiar da mãe $e^{(5,7)}$.

Em relação ao aleitamento materno, defendido pelas organizações envolvidas com a saúde da criança, os participantes deste estudo enfatizaram que a não realização da visita domiciliar em tempo ideal traz comprometimentos a esssa prática, colaborando, entre outros, para o desmame precoce que possivelmente afetará na saúde e desenvolvimento ao longo da vida do RN.

Se essa criança chega na primeira semana e a mãe não está conseguindo amamentar, lógico que na maternidade ela sai já amamentando, mas muitas vezes a dificuldade é grande na casa, no domicílio, especialmente se tiver uma avó, uma mãe, uma pessoa que está ali que possa dificultar, diz dê mingau, dê outro leite e a própria mulher insegura compra um leite mesmo e dá. (E2)

A amamentação a gente também tem que está de olho, tem mãe que ela segue rigorosamente a amamentação exclusiva, tem mãe que já sai da maternidade querendo dar uma alimentação complementar. (E3)

Se você não trabalhar na primeira semana e começar a dar água ou algum composto, a criança se acostuma logo [...] se você fizer uma visita a uma puérpera, agora pode não acontecer, depois de 10 dias, 15 dias, que ela já tiver dado qualquer outro complemento, fica muito mais difícil de você conquistar essa mulher a deixar. (E7) 
A amamentação foi um aspecto bastante enfatizado pelos enfermeiros neste estudo, sobretuto no que se refere à inserção de leites artificiais de forma parcial e até total substituição do leite materno, especialmente quando a visita domiciliar era tardia e quando havia a presença de familiares participando dos cuidados ao RN. Diante disso, enfatiza-se a importância da assistência ao binômio mãebebê seguir as recomendações do MS para a melhor detecção de fatores que influenciam nos cuidados básicos ao RN, sobretudo no que se refere ao aleitamento materno que é uma prática recomendada para ser exclusiva até os seis meses de idade, e, quando implementada, contribui significativamente para redução da morbidade infantil (7).

Os enfermeiros explicitaram preocupação quanto ao manejo com o RN nesse período de vida, porém, algumas ações, a exemplo da detecção de fatores de risco, tanto ambientais como biológicos, que são importantes na prevenção de agravos, só foram mencionadas no relato de um enfermeiro:

Eu vejo a forma como ela (a criança) está sendo acolhida, [...] pode ter a questão de drogas, de álcool, então a gente tem que ver os riscos que essa criança pode estar correndo, diante do âmbito familiar. (E2)

Esse aspecto relatado por apenas um participante do estudo demonstra fragilidade na assistência dos enfermeiros ao RN, uma vez que foi minimamente abordada a preocupação em investigar os fatores de risco, levando-nos a inferir que há escassez de conhecimento e preparo na prestação de cuidados integrais a essa população e, por ser período de vulnerabilidades, as ações de saúde deveriam ser mais incisivas e efetivas para uma real diminuição das taxas de mortalidade.

Além dos cuidados básicos ao RN, deve haver a identificação de fatores de risco que o mesmo estará exposto na dinâmica familiar como violência doméstica, risco a acidentes, usuário de álcool e drogas no domicílio e acolhimento do neonato ${ }^{(7)}$. Estudo realizado em Curitiba ${ }^{(15)}$ acerca das vulnerabilidades para o desenvolvimento infantil, destacou a dependência química, em especial dos pais, como fator que afeta diretamente o desenvolvimento infantil, uma vez que não há a criação de laços afetivos e ações de proteção à criança.

Existem algumas preocupações acerca do seguimento de recomendações das práticas clínicas em todo o mundo, especialmente por serem baseadas em evidências científcas que visam a melhoria da saúde. Nesse contexto, as diretrizes têm papel importante na redução de morbidades e, por serem norteadas por políticas públicas e embasadas teoricamente, devem ser seguidas, no intuito de guiar as ações dos profissionais durante a primeira visita domiciliar visando assistir a mãe e o neonato de forma integral.

Em se tratando da promoção e avaliação do estado de saúde, bem como de orientações que contemplam a saúde materna no período puerperal, foi possível identificar uma contradição nos discursos dos entrevistados, uma vez que estes se referem à visita domiciliar como "visita puerperal", no entanto, apenas um enfermeiro prestou assistência à mãe, enquanto os demais deram enfoque às orientações sobre o planejamento familiar.

A questão da mãe também, não é só pensar na criança, mas pensar na mãe também; como é que está a questão do lóquios no pós-parto; Como é que ela está vivenciando, porque tem a questão da depressão; Como está a situação geral da família, principalmente dela, que a gente tem que ter atenção com a mãe; Como é que ela está se comportando, se portando diante desse bebê, principalmente se for mãe de primeira viagem. (E2)

Orientar ela (a puérpera) sobre o anticoncepcional que ela vai tomar com 42 dias de resguardo. (E9)

É importante destacar que apesar deste estudo se referir às ações realizadas ao RN, não se pode desvincular do binômio mãe-bebê uma vez que o neonato é dependente dessa, sendo, portanto, imprescindível a avaliação dos aspectos biológicos, psíquicos e sociais maternos. Assim, no sentido de cumprir os pressupostos recomendados pela PSSI, é primordial que o profissional esteja atento às condições que interferem também no estado de saúde da mãe, isto porque a saúde da criança também estará em risco caso a mãe não esteja bem com sua saúde, devendo, então, ser foco da atenção dos profissionais nos primeiros dias de puerpério ${ }^{(5)}$.

É importante enfatizar que a escassez de cuidados preconizados acerca da saúde materna demonstrada neste estudo está em concordância com outro cujos achados apontam falhas na assistência à puérpera durante a visita domiciliar, uma vez que esta não é devidamente avaliada e suas queixas nem sempre são ouvidas pelos enfermeiros ${ }^{(16)}$. Outra orientação importante não explicitada pelos enfermeiros em suas ações são os sinais de perigo que os profissionais devem atentar quando assistirem uma puérpera, sendo eles: sangramento vaginal, dor de cabeça, convulsão, transtornos visuais, dor abdominal, disúria, febre, perdas vaginais, dificuldade respiratória e cansaço ${ }^{(7)}$. Isso sugere que a atuação desses profissionais na APS estão aquém 
do que recomenda a literatura, levantando a necessidade de melhor investigar os motivos que levam a essa prática inconsistente, para subsidiar posterior tomada de decisão no que concerce a medidas reparadoras.

Merece destaque também o papel da família para o incentivo na adaptação da puérpera à nova dinâmica familiar e no empoderamento da mãe assim como auxílio nos cuidados ao RN em atividades diárias no domicílio. Sob a mesma perspectiva, um estudo realizado no Japão com jovens primíparas identificou que a presença de problemas familiares e dificuldade de comunicação com o parceiro influenciam negativamente a confiança da mãe diante da maternidade ${ }^{(17)}$.

Dessa forma, dentre as ações dos enfermeiros, inclui-se um olhar atento para a detecção de fatores de risco ao RN e à puérpera, considerando os físicos e emocionais da mãe, e o apoio familiar para a tomada de decisão e direcionamento da melhor estratégia de cuidado ao binômio(15).

Em se tratando ainda das ações dos enfermeiros, foi destacado a importância da visita domiciliar em incentivar a mãe em levar o RN para a puericultura, possibilitando a continuidade do cuidado.

E outra coisa importante que eu acho que é bom relatar, quando a gente vai, a gente já incentiva essa mãe a vir para a puericultura e isso que é importante também porque a gente já forma esse link, né, dela vir à consulta e ficar vindo. (E2)

Nesse contexto, destaca-se que a construção do vínculo se inicia no pré-natal e tem papel fundamental para o acompanhamento da criança, uma vez que a mãe se sente mais confiante com o enfermeiro e comparece mais ao serviço para a puericultura. A continuidade da assistência possibilita a avaliação do crescimento e desenvolvimento da criança, detecção precoce de problemas, orientação e encaminhamento para imunização ${ }^{(18)}$. Ademais, estudo(19) destaca que o vínculo é fundamental para a realização da consulta de puericultura na APS, por proporcionar também estreitamento de laços e interação efetiva e afetiva com a família.

Os discursos dos enfermeiros permitiram também identificar fragilidades nas ações devido falta de conhecimento. Em contrapartida, o relato a seguir também revela a carência de ações de educação em saúde para esses profissionais.

Falta para a gente esse contato com a academia [...] eu acho que a atenção básica tá muito precária nesse sentido de tá com os trabalhadores dando treinamento, atualizações entendeu? (E8)
Ante o exposto, destaca-se a necessidade de efetivação de educação permanente com os profissionais da atenção primária, no que se refere à PSSI, para que estes aprimorem seu processo de trabalho e implementem as ações específicas do cuidado à saúde materna e infantil com segurança e baseadas nas diretrizes de saúde, tendo em vista que foram identificadas fragilidades de conhecimento dos enfermeiros acerca das políticas de saúde vigentes voltadas para a assistência à puérpera e ao RN. Estudo realizado em Cuiabá - MG concluiu que apenas metade dos enfermeiros se sentem aptos a realizar a assistência às puérperas e aos $\mathrm{RN}^{(20)}$, corroborando com o presente estudo a necessidade de capacitações dos profisisonais da ESF.

\section{口 CONSIDERAÇÕES FINAIS}

A implementação da Primeira Semana Saúde Integral no cuidado ao RN é importante para se realizar uma assistência integral ao neonato e à puérpera. Assim, foi possível perceber neste estudo potencialidades nas ações dos enfermeiros como as orientações sobre cuidados básicos, promoção do aleitamento materno, empoderamento da mulher durante o pré-natal e vínculo do profissional com a família e, embora necessitem ser mais aprofundadas, representam potencial para melhorar a assistência ao binômio mãe-bebê.

No entanto, foram identificadas fragilidades nas ações dos enfermeiros da ESF referentes à assistência a essa população, relacionadas ao não cumprimento do tempo ideal para a realização da primeira visita ao RN conforme recomendado pelo MS, ausência de observação dos fatores de risco para a saúde do neonato e lacuna na assistência às puérperas, que são condizentes com estudos nacionais, levando-nos a questionar se a formação em enfermagem está sendo adequada às demandas de saúde da população usuária dos serviços da APS.

Com isso, surge a necessidade de uma ação conjunta de Educação Permanente em Saúde com profissionais das USF e gestores, a fim de capacitar esses profissionais para o cuidado à puérpera e ao RN, especialmente no âmbito APS que é norteadora do cuidado a essa população, no sentido de contemplar as diretrizes preconizadas para a redução da morbimortalidade infantil, como também, fornecer um cuidado integral em saúde a essa população. Também se faz necessário novos estudos que abordem as práticas dos profissionais da USF, principalmente sob a ótica da PSSI e a percepção das puérperas diante dessas ações, de forma a tornar mais evidente essa estratégia de cuidado e, assim, a contribuir para o acompanhamento do RN o mais precoce possível e para a promoção do crescimento e desenvolvimento. 
Consideramos como limitação do estudo o tamanho da amostra que apresentou número reduzido devido à falta de disponibilidade dos enfermeiros da USF do Distrito Sanitário que foi alvo da pesquisa.

\section{QREFERÊNCIAS}

1. United Nations (US). The Millennium Development Goals report 2015. New York: United Nations; 2015.

2. United Nations Population Fund (US). The state of the world's midwifery 2014: a universal pathway: a woman's right to health. New York: United Nations; 2014.

3. United Nations Statistics Division (US). Millennium Development Goals Indicators [Internet]. New York: UN; c2014-2016 [cited 2016 May 31]. Available from: http://mdgs.un.org/unsd/mdg/Data.aspx.

4. Lansky S, Friche AAL, Silva AAM, Campos D, Bittencourt SDA, Carvalho ML, et al. Birth in Brazil survey: neonatal mortality profile, and maternal and child care. Cad Saúde Pública. 2014;30(Suppl. 1):S1-S15.

5. Ministério da Saúde (BR). Agenda de compromissos para a saúde integral da criança e redução da mortalidade infantil. Braślila (DF): MS; 2004.

6. Ministério da Saúde (BR). Portaria no 1.130, de 5 de agosto de 2015. Institui a Política Nacional de Atenção Integral à Saúde da Criança (PNAISC) no âmbito do Sistema Único de Saúde (SUS). Diário Oficial da União [da] República Federativa do Brasil. 2015 ago 06;152(149 Seção 1):37-9.

7. Ministério da Saúde (BR). Saúde da criança: crescimento e desenvolvimento. Brasília (DF): MS; 2012. Cadernos de Atenção Básica no 33.

8. Sitrin D, Guenther T, Waiswa P, Namutamba S, Namazzi G, Sharma S, et al. Improving newborn care practices through home visits: lessons from Malawi, Nepal, Bangladesh, and Uganda. Glob Health Action. 2015;8:23963.

9. Souza MHN, Gomes TNC, Paz EPA, Trindade CS, Veras RCC. Estratégia acolhimento mãe-bebê: aspectos relacionados à clientela atendida em uma unidade básica de saúde do município do Rio de Janeiro. Esc Anna Nery. 2011;15(2):671-7.
10. Minayo MCS. 0 desafio do conhecimento: pesquisa qualitativa em saúde. 14. ed. São Paulo (SP): Hucitec; 2014.

11. Nascimento JS, Costa LMC, Santos RM, Anjos DS. Visitas domiciliares como estratégias de promoção da saúde pela enfermagem. Rev Bras Promoc Saúde. 2013;26(4):513-22.

12. Rodrigues TMM, Vale LMO, Leitão RAR, Silva RMO, Rocha SS, Pedrosa JIS. A visita domiciliar do enfermeiro à puérpera e ao recém-nascido. Rev Interd NOVAFAPI. 2011;4(2):21-6.

13. Souza RS, Ferrari RAP, Santos TFM, Tacla MTGM. Atenção à saúde da criança: prática de enfermeiros da saúde da família. REME. 2013;17(2):331-9.

14. Shorey S, Chan SWC, Chong YS, He HG. Maternal parental self-efficacy in newborn care and social support needs in Singapore: a correlational study. I Clin Nurs. 2014;23(15-16):2272-82.

15. Silva DI, Maftum MA, Mazza VA. Vulnerabilidade no desenvolvimento da criança: influência dos elos familiares fracos, dependência química e violência doméstica. Texto Contexto Enferm. 2014;23(4):1087-94.

16. Mazzo MHSN, Brito RS, Santos FAPS. Atividades do enfermeiro durante a visita domiciliar pós-parto. Rev Enferm UERJ. 2014;22(5):663-7.

17. Maehara K, Mori E, Tsuchiya M, Iwata H, Sakajo A, Ozawa H, et al. Factors affecting maternal confidence among older and younger Japanese primiparae ate one month post-partum. Jpn J Nurs Sci. 2016;13(4):424-36.

18. Silva LLB, Feliciano KVO, Oliveira LNFP, Pedrosa EN, Corrêa MSM, Souza AI. Cuidados prestados à mulher na visita domiciliar da "Primeira Semana de Saúde Integral". Rev Gaúcha Enferm. 2016;37(3):e59248.

19. Reichert APS, Rodrigues PF, Albuquerque TM, Collet N, Minayo MCS. Vínculo entre enfermeiros e mães de crianças menores de dois anos: percepção de enfermeiros. Cienc Saúde Coletiva. 2016;21(8):2375-82.

20. Oliveira DC, Mandú ENT, Corrêa ACP, Tomiyoshi JT, Teixeira RC. Estrutura organizacional da atenção pós-parto na Estratégia Saúde da Família. Esc Anna Nery. 2013;17(3):446-54.

\section{- Autor correspondente:}

Daniele Beltrão de Araújo Lucena E-mail: danibeltraoal@gmail.com
Recebido: 17.04.2017

Aprovado: 27.07.2017 